\title{
Comparison of ICU transfusion practice (with and without autologous transfusion) in a cardiac surgery population
}

Van Nieuwenhove S. MD, Verbeke J. MD, De Neve N. MD, De Decker K. MD. Department of Intensive care, OLV hospital, Aalst, Belgium

Background

Several trials concluded that postoperative transfusion increases the risk for complications in cardiac surgery patients ${ }^{(1-2)}$. Although this idea was challenged by other reports, it has led to the development of cell saving devices that collect autologous blood and - after washing of the red blood cells - deliver an end product with a high hematocrit.

\section{Objectives}

For several years, some of our surgeons used the CardioPAT receiving autologous blood and the group that did not. The device (Haemonetics, Braintree, Massachusetts, USA), but due to incidence of reinterventions was $8.73 \%$ in the cell saved group vs production problems and the company takeover, autologous $16.52 \%$ in the non-cell saved group ( $p=0.005)$. There was also a transfusion was reduced in 2016 and finally stopped early January statistically significant change in severity of acute kidney injury 2017. In order to look at the impact of this management change, we $(26.75 \%$ vs $55.13 \%$ rise in post- vs preoperative serum creatinine evaluated our transfusion numbers and several other outcome data.

Materials and methods

We compared the transfusion data from the previous years (20152016) with the data from 2017, in which the device was no longer used. With regard to transfusion data we looked at the amount of blood products used and the percentage of patients receiving them in the postoperative phase. Furthermore, we made a comparison of these data between the group receiving autologous transfusion and the group that did not. Finally we also describe several other outcome parameters (reintervention rate, new onset of atrial fibrillation, acute kidney injury, length of ICU stay and mortality).

\section{Results}

The number of procedures in 2015, 2016 and 2017 was 674, 640 and 456 (untill August $31^{\text {st }}$ ) respectively. A total amount of 351 and 186 units of cell saved blood were retransfused in the first two years, correlating with 82.9 and 33.6 liters of autologous blood. The percentage of patients receiving allogeneic red blood cells increased (6\%) in parallel with the reduction of cell saving, but the amount of blood products did not (table 1). The ICU length of stay did not change over the years (3.05, 3.08 and 3.01 days respectively), nor did the mortality.

We then divided all patients receiving blood products in two groups, one group with and another one without the retransfusion of autologous blood. Transfusion data are presented in table 2.

Previous reports on the use of the CardioPAT device were contradictory. A large Italian trial (3), investigating intra- and postoperative autotransfusion, showed a significant reduction in exposure to allogeneic RBC's. Complications were also less frequent and the use of CardioPAT appeared to be safe.

A more recent Dutch trial (4) did not show reduced transfusion requirements compared to intraoperative cell salvage alone and showed higher CK levels. Transfusion is significantly higher in the non-cell saved group. The data are somewhat higher than the percentages mentioned in the two previous trials.

When comparing our two groups of transfused patients, we noticed differences in various outcome parameters between the group $(p<0.0001)$, and the length of stay on the ICU (3.79 vs 5.76 days, $p<0.0001)$. There was no statistical significant difference between the incidence of postoperative new onset atrial fibrillation $(16.71 \%$ vs $18.75 \%, p=0.5)$ nor mortality $(3.99 \%$ vs $6.7 \%, p=0.17)$ (table 3$)$.

Table 1

\begin{tabular}{|l|c|c|c|}
\cline { 2 - 4 } \multicolumn{1}{c|}{} & packed cells & fresh frozen plasma & platelets \\
\hline 2015 & $574(21)$ & $289(12)$ & $148(10)$ \\
\hline 2016 & $601(25)$ & $290(13)$ & $173(11)$ \\
\hline 2017 & $353(27)$ & $118(10)$ & $65(9)$ \\
\hline
\end{tabular}

Total number of transfused blood products (\% of transfused patients)

Table 2

\begin{tabular}{|l|c|c|}
\cline { 2 - 3 } \multicolumn{1}{c|}{} & CS & no CS \\
\hline Number of patients & 401 & 224 \\
\hline Total amount of packed cells given & 403 & 719 \\
\hline Packed cells/patient & 1 & 3,21 \\
\hline Total amount of fresh frozen plasma given & 232 & 309 \\
\hline Fresh frozen plasma/patient & 0,58 & 1,38 \\
\hline Total amount of platelets given & 130 & 178 \\
\hline Platelets/patient & 0,32 & 0,79 \\
\hline
\end{tabular}

Table 3

\begin{tabular}{|l|c|c|}
\cline { 2 - 3 } \multicolumn{1}{|c|}{} & CS & no CS \\
\hline Reintervention (\%) & 8,73 & 16,52 \\
\hline New onset atrial fibrillation (\%) & 16,71 & 18,75 \\
\hline \% rise in creatinine post- vs preoperatively & 26,75 & 55,13 \\
\hline LOS ICU (days) & 3,79 & 5,76 \\
\hline Deaths (\%) & 3,99 & 6,7 \\
\hline
\end{tabular}

\section{Conclusions}

Abandoning autologous postoperative transfusion did not result in higher transfusion rates of allogeneic blood. The number of patients receiving RBCs increased slightly, but the total amount of blood products was not statistically significant.

There was a significant difference in the group of transfused patients when comparing patients that received autologous retransfusion vs the ones that didn't concerning length of stay on the ICU, reintervention rates and the severity of acute kidney injury.

\section{References}

(1) Horvath $\mathrm{K}$, Acker M, Chang $\mathrm{H}$ et al. Blood transfusion and infection after cardiac surgery. Ann thorac surg 2013;95;2194-201.

(2) Kilic A, Whitman G. Blood transfusions in cardiac surgery: indications, risks, and conservation strategies. Ann thorac surg 014;97;726-34.

(3) Weltert L, Nardella S, Rondinelli MB et al. Reduction of allogeneic red blood cell usage during cardiac surgery by an integrated intra- and postoperative blood salvage strategy: results of a randomized comparison. Transfusion 2013;53;790-797.

(4) Vermeijden WJ, Hagenaars JAM, Scheeren TWL et al. Additional postoperative cell salvage of shed mediastinal blood in cardiac surgery does not reduce allogeneic blood transfusions: a cohort study. Perfusion 2016, Vol. 31(5),384-390.

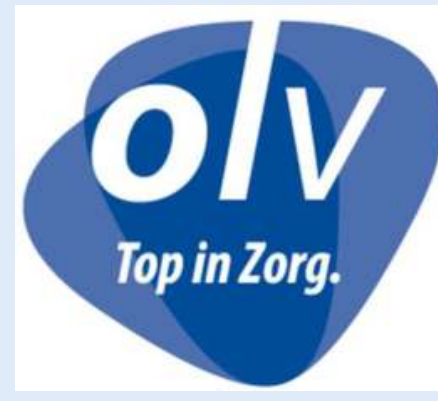

